\title{
Comparison of a Cancer Family History Collection and Risk Assessment Tool - ItRunsInMyFamily - with Risk Assessment by Health-Care Professionals
}

\author{
Jordon B. Ritchie ${ }^{a}$ Brandon M. Welcha, ${ }^{a}$ Caitlin G. Allen ${ }^{c}$ Lewis J. Frey ${ }^{a}$ Heath Morrison ${ }^{a}$ \\ Joshua D. Schiffman ${ }^{d}$ Alexander V. Alekseyenko ${ }^{a}$ Brian Dean ${ }^{e}$ Chanita Hughes Halbert ${ }^{a, b}$, $f$ \\ Cecelia Bellcross ${ }^{9}$

\begin{abstract}
aDepartment of Public Health Sciences, Medical University of South Carolina, Charleston, SC, USA; bHollings Cancer Center, Medical University of South Carolina, Charleston, SC, USA; 'Department of Behavioral, Social, and Health Education Sciences, Emory University, Rollins School of Public Health, Atlanta, GA, USA; dOncological Sciences, Huntsman Cancer Institute at the University of Utah, Salt Lake City, UT, USA; ${ }^{\circ}$ Computer Science, Clemson University, Clemson, SC, USA; fDepartment of Psychiatry and Behavioral Sciences, Medical University of South Carolina, Charleston, SC, USA; ${ }^{9}$ Department of Human Genetics, Emory University, Atlanta, GA, USA
\end{abstract}

\section{Keywords}

Hereditary cancer · Risk assessment · Family health history ·

Clinical practice guidelines

\begin{abstract}
Introduction: Primary care providers (PCPs) and oncologists lack time and training to appropriately identify patients at increased risk for hereditary cancer using family health history (FHx) and clinical practice guideline (CPG) criteria. We built a tool, "ItRunsInMyFamily" (ItRuns) that automates FHx collection and risk assessment using CPGs. The purpose of this study was to evaluate ItRuns by measuring the level of concordance in referral patterns for genetic counseling/testing (GC/GT) between the CPGs as applied by the tool and genetic counselors (GCs), in comparison to oncologists and PCPs. The extent to which non-GCs are discordant with CPGs is a gap that health information technology, such as ItRuns, can help close to facilitate the identification of individuals at risk for hereditary cancer. Methods: We curated 18 FHx cases and surveyed GCs and non-GCs (oncologists and PCPs) to assess concordance with ItRuns CPG criteria for referring patients for GC/GT. Percent agreement was used to describe concordance, and
\end{abstract}

karger@karger.com www.karger.com/phg

Karger $\stackrel{\text { ' }}{5}$

BOPEN ACCESS
(C) 2021 The Author(s)

Published by S. Karger AG, Basel

This is an Open Access article licensed under the Creative Common Attribution-NonCommercial-4.0 International License (CC BY-NC) (http://www.karger.com/Services/OpenAccessLicense), applicable to the online version of the article only. Usage and distribution for commercial purposes requires written permission. logistic regression to compare providers and the tool's concordance with CPG criteria. Results: GCs had the best overall concordance with the CPGs used in ItRuns at $82.2 \%$, followed by oncologists with $66.0 \%$ and PCPs with $60.6 \%$. GCs were significantly more likely to concur with CPGs (OR $=4.04,95 \% \mathrm{Cl}=$ 3.35-4.89) than non-GCs. All providers had higher concordance with CPGs for FHx cases that met the criteria for genetic counseling/testing than for cases that did not. Discussion/ Conclusion: The risk assessment provided by ItRuns was highly concordant with that of GC's, particularly for at-risk individuals. The use of such technology-based tools improves efficiency and can lead to greater numbers of at-risk individuals accessing genetic counseling, testing, and mutation-based interventions to improve health.

(c) 2021 The Author(s)

Published by S. Karger AG, Basel

\section{Introduction}

Between 5 and $10 \%$ of all cancers are hereditary [1]. Patients identified as at risk for hereditary cancer should be offered cancer genetic counseling and, if appropriate,
Correspondence to:

Jordon B. Ritchie, ritchiej@musc.edu 
genetic testing [2]. Identification of a high-penetrance cancer gene mutation allows for screening and prevention strategies to mitigate cancer risks for the patient and at-risk relatives [3-9]. Unfortunately, many patients at risk for hereditary cancer are being missed [10-13]. For example, a study of the greater London population concluded that only $10.9 \%$ of BRCA carriers have been identified [14]. Another study estimated that fewer than one-in- 5 patients in the USA with a history of breast or ovarian cancer who meet clinical practice guidelines (CPGs) have received genetic testing. Additionally, most had not discussed testing with their provider [15]. Identification rates of individuals at risk for other hereditary cancer gene mutations (e.g., BRIP1, PALB2, and ATM) are likely lower.

CPGs published by the National Comprehensive Cancer Network (NCCN), the American College of Medical Genetics and Genomics (ACMG), and others have been developed to assist providers in more appropriately referring their patients for cancer genetic consultation based on family health history (FHx). However, research has shown that CPGs have little effect on improving clinical practice and decision-making [16]. Regarding hereditary cancer, there are several reasons why at-risk patients are not identified or referred for cancer genetic consultation. An incomplete or inaccurate FHx makes it difficult to assess hereditary cancer risk, and collecting complete and accurate FHx can be a tedious, time-consuming task [17]. Providers, especially those in primary care specialties, typically are limited to short patient visits and lack training and confidence in collecting and assessing FHx for hereditary cancer risk $[18,19]$. Even once FHx is collected, applying hereditary cancer CPGs can be difficult as they are relatively lengthy and complicated $[20,21]$. Additionally, providers are not reimbursed for time spent collecting and assessing FHx [22]. The inconsistent application of CPGs can result in missed referrals to genetic counseling and testing.

ItRuns is an informatics tool that collects FHx using a chatbot and evaluates it using CPGs, ontologies, and web services $[23,24]$. ItRuns has been used to collect and assess 4,915 patient-entered family histories of cancer [25]. Once patients enter their FHx through the ItRuns chatbot, they receive a PDF report summarizing their FHx and whether they meet the criteria for genetic counseling/ testing based on the ItRuns CPGs used. Research has shown that patients prefer chatbots over web forms or other methods when collecting FHx, resulting in higher rates of participation [26, 27]. Chatbots are programs that simulate a conversation-based data collection method to provide a more natural data collection experience.
Collecting FHx before patients meet with their providers saves time during an office visit and results in more complete, standardized, and accurate FHx information [17]. Web technology such as ItRuns provides patients and providers easy access to $\mathrm{FHx}$ collection and evaluation to determine whether a patient meets the thresholds that warrant a referral for cancer genetic consultation $[28,29]$. CPG criteria selected to implement in ItRuns were represented in an ontology-driven rule system and reviewed by a cancer genetic counselor and a clinical oncologist [24]. Technology-assisted FHx collection and risk assessment is a promising approach that identifies patients at risk for hereditary cancer who would otherwise potentially be missed.

The purpose of this study was to evaluate the accuracy of the CPG-guided ItRuns risk assessment by comparing it to pedigree risk assessment performed by genetic counselors (GCs). We also evaluated non-GC (oncologists and primary care providers [PCPs]) risk assessment for additional comparative data. We predicted that genetic counselors' assessment of risk status would be most concordant with ACMG and NCCN CPG criteria implemented by the ItRuns tool, relative to PCPs and oncology providers. Examining the concordance between an informatics approach for obtaining and assessing FHx of hereditary disease risk is an important step in validating a technology solution for identifying patients who are appropriate candidates for genetic counseling and testing.

\section{Methods}

Study Design

To compare concordance among GCs, oncologists, and PCPs, we developed 18 fictitious FHx cases with patterns of affected probands and family members. We designed these cases so that half would meet ACMG and NCCN criteria for genetic counseling/ testing referral implemented by the ItRuns tool and half would not. The probands meeting the criteria depended on cancer types, ages of onset, ancestry, and number of family members with cancer. These cases were then run through the ItRuns system by a study team member to obtain referral recommendations. The family cancer history and associated pedigrees (see Fig. 1) for every case were shown to GCs, oncologists, and PCPs in a survey to determine each group's respective referral patterns for whether the individual should receive genetic counseling/testing. Results were compared and analyzed for consistency. This study was reviewed and approved as exempt by the IRB at the Medical University of South Carolina.

\section{Data Collection}

The survey, which ran from January 15 to February 8, 2021, was sent to GCs through the National Society of Genetic Counselors' Cancer Special Interest Group listserv. Oncologists and PCPs were 
Executive summary

Proband is a 60 year old female with

- No reported personal history of cancer

- European ancestry

- No reported personal gene mutation

- No reported family gene mutation history

- Family history of breast cancer pancreatic cancer

Family cancer history

Breast cancer

- Maternal aunt diagnosed at 52 years old

Pancreatic cancer

- Father diagnosed at 74 years old

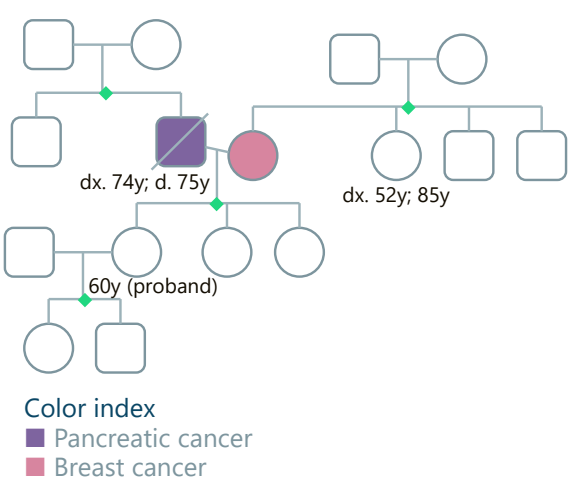

Fig. 1. An example FHx case.

gives an example of an FHx case contained in the survey, and the complete survey can be viewed here: https://redcap.musc.edu/ surveys/?s=DDRWE9WL8T.

\section{Data Analysis}

We calculated percent concordance of each group's assessment with the CPG referral recommendations as implemented by ItRuns. Logistic regression was used to provide adjusted assessment of concordance, and to compare between GCs and non-GCs. The analysis was performed in $\mathrm{R}$ version 3.5.2 using the generalized linear models library. Models were built to compare overall concordance, concordance for cases that met the CPG criteria implemented by ItRuns for referral, and concordance for cases that did not meet the criteria. Models were built to compare GCs versus non-GCs and to compare GCs versus oncologists and versus PCPs specifically. All models were adjusted for the specific case. implemented by ItRuns and half did not. The order of the cases in the survey was randomized by assigning each FHx case a number and using a randomization function to determine the order in which the cases would be presented. Participants reviewed the pedigrees and provided an assessment of whether the individual should or should not be referred for genetic counseling/testing.

\section{FHx Cases}

FHx cases were constructed manually by the authors (J.B.R. and B.M.W.) and reviewed by a board-certified genetic counselor (C.B.). The cases included an executive summary section, a family cancer history section, and a 3-generation cancer pedigree. The executive summary gives an overview of the proband including their age, personal and family cancer history, age of onset for each cancer in their family, ancestry, and any personal or familial genetic mutations. The FHx includes each family member with a cancer diagnosis and associated age of onset. The 3-generation pedigree includes all relatives in the family, color coding for different cancers, age, age of death, and age of diagnosis for affected relatives. The cases that meet the criteria focused on those for which single instances of breast, ovarian, or colorectal cancer with specific age onset were sufficient to trigger a recommendation. A few of these cases had other cancers present in the family. Cases designed to not meet the criteria included cancer, but of a later age of onset or type not consistent with the CPG referral recommendations. Figure 1

\section{Results}

\section{CPG Results}

The FHx tool ItRuns was designed to be consistent with ACMG/NCCN CPGs for all 18 cases. Overall concordance with the ItRuns CPG recommendations for all provider types was $88.5 \%$ for cases that met the criteria for referral, $64.7 \%$ for cases that did not, and $76.6 \%$ overall. All providers had higher concordance for FHx cases that met the criteria versus those that did not (Fig. 2). For cases that met the criteria for genetic counseling/testing, providers' concordance with the ItRuns CPG assessment ranged from $72.1 \%$ (case 12 ) to $98.7 \%$ (case 18 ). Among cases that did not meet the criteria, providers had lower and more varied rates of concordance ranging from $32.7 \%$ (case 5) to $100 \%$ (case 1). All providers had $100 \%$ concordance for only one of the 18 cases - case 1 - where the proband has a maternal grandmother diagnosed with lung cancer at 77 years old and no referral would be recommended. 
Fig. 2. Overall concordance with CPGs for all providers. Red indicates providers recommended referral, and gray indicates providers did not recommend referral. The chart in (a) shows cases that did not meet the criteria; therefore, gray bars represent concordance with the CPGs. The chart in (b) shows cases that met the criteria; therefore, red bars represent concordance with the CPGs. Cases are sorted in increasing order by GCs' concordance with CPGs. CPGs, clinical practice guidelines; GCs, genetic counselors.

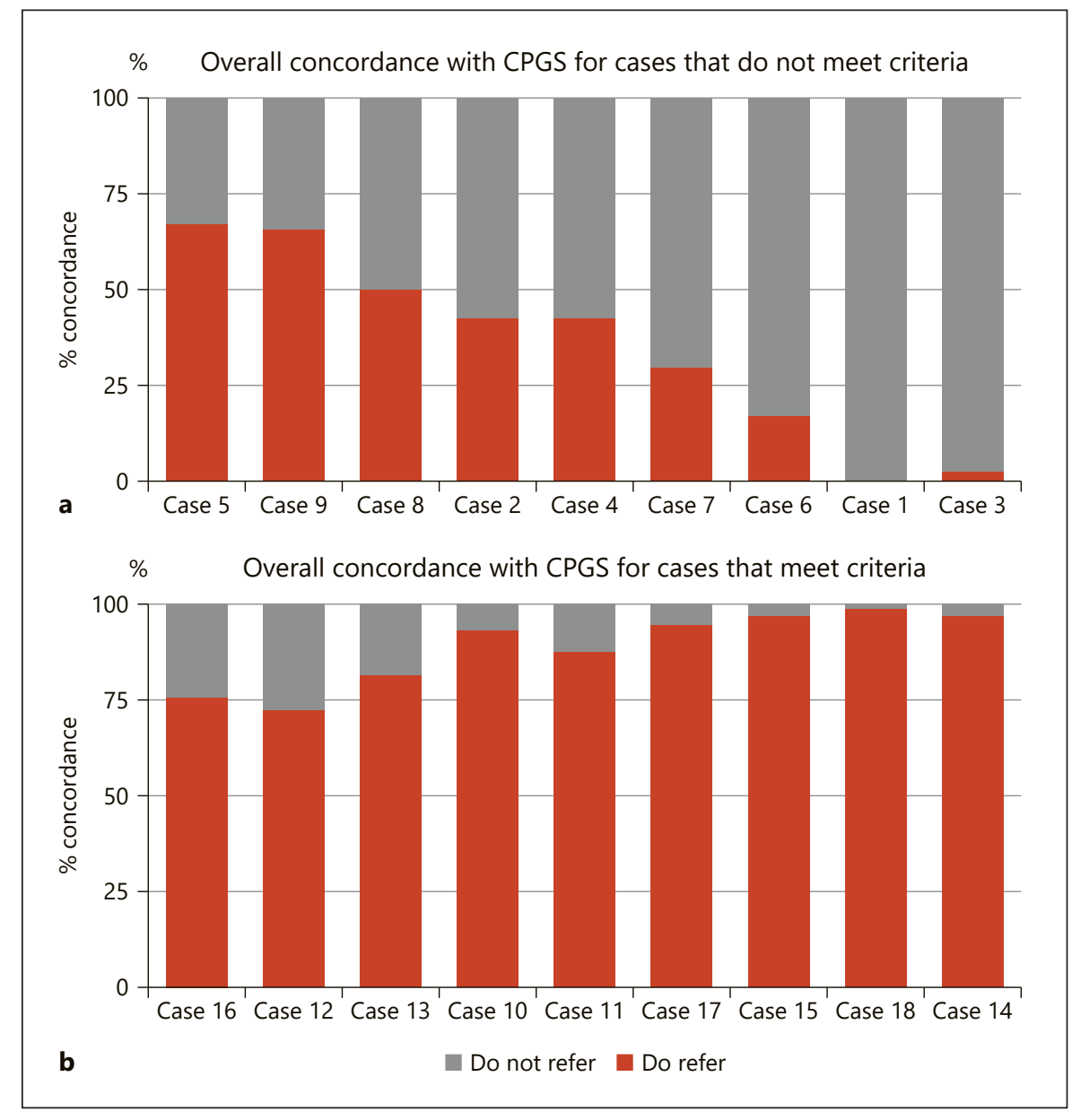

\section{Demographics}

A total of 159 (70.4\%) GCs and 67 (29.6\%) non-GCs participated in the survey for a total of 226 participants. Of the 67 non-GCs, 34 (50.8\%) were identified as oncologists, $27(40.3 \%)$ were identified as PCPs, and $6(8.9 \%)$ were identified as "other." The 6 who were identified as "other" included 2 radiologists, an acute care nurse practitioner, a molecular genetic pathologist, a breast surgeon, and a hospitalist physician assistant. GCs and oncologists averaged 8.5 and 17.5 years of experience, respectively. PCPs and all others averaged 12.3 years of experience. Of the 226 participants, 175 (77.4\%) were identified as female, 49 (21.7\%) were identified as male, and $2(0.9 \%)$ preferred not to say. GCs were the least balanced by sex, with 150 (94.3\%) participants being identified as female, 7 (4.4\%) identified as male, and 2 (1.3\%) preferring not to say. Oncologists were similarly but inversely unbalanced by sex, with 27 (79.4\%) participants being identified as male and 7 (20.6\%) as female. PCPs were relatively even, with 18 (54.6\%) being identified as female and $15(45.4 \%)$ as male.

Comparison of Cancer Family History

Risk Assessments
Genetic Counselor's Concordance with CPGs

GCs' overall concordance with CPGs was $82.2 \%, 97.6 \%$ for cases that met the CPG criteria implemented by ItRuns, and $66.7 \%$ for cases that did not. Out of all provider types, GCs had the closest concordance with CPGs. For cases that did not meet the criteria, GCs' concordance ranged from $35.2 \%$ (case 5) to $100 \%$ (cases 1 and 3 ) (Fig. 3a). For cases that met the criteria, GCs' concordance ranged from $93.7 \%$ (cases 12 and 16 ) to $99.4 \%$ (case 18) (Fig. 3b). Genetic counselors had over $93 \%$ concordance for all cases that met the ItRuns CPG criteria and over $98 \%$ concordance for two-thirds of these (Table 1). For 2 of the 9 cases that did not meet the CPG criteria, GCs scored $100 \%$ concordance.

\section{Non-GC's Concordance with CPGs}

Non-GCs less consistently suggested referral than the CPGs at $63.4 \%$ overall, $66.7 \%$ for cases that met the CPG criteria implemented by ItRuns, and $60 \%$ for cases that did not meet the criteria. Oncologists had overall concordance 
Fig. 3. Concordance between CPGs and GCs (gray line), Onc (red line) and PCPs (yellow line) for cases that do not meet the CPG criteria implemented by ItRuns (a) and cases that do meet the CPG criteria. GCs had better concordance with CPGs than all others, especially for cases that met the criteria (b). For cases that did not meet the CPG criteria, all providers showed considerable discordance. CPGs, clinical practice guidelines; GCs, genetic counselors; ItRuns, ItRunsInMyFamily; Onc, oncologist; PCPs, primary care providers.

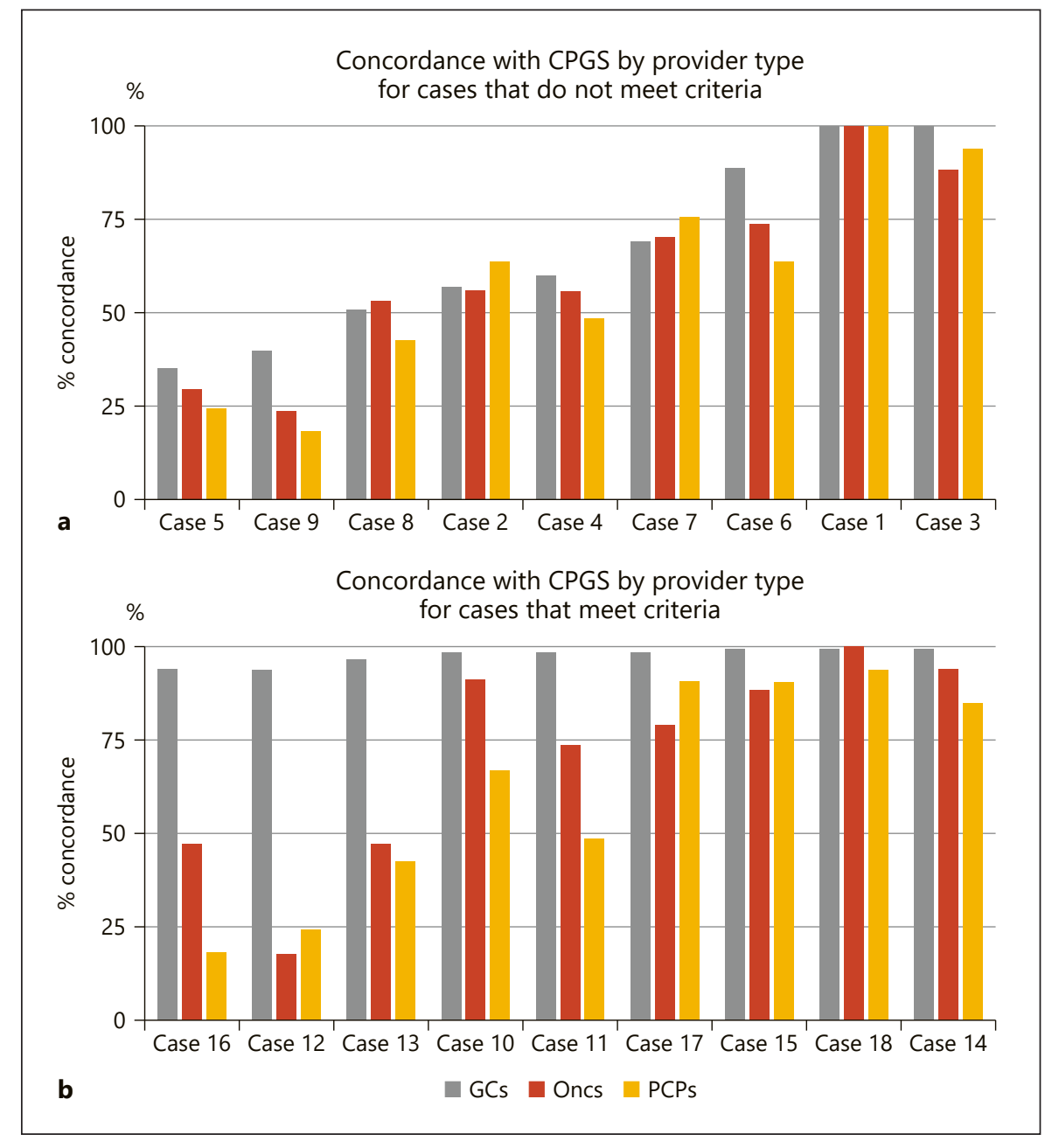

with CPGs $66 \%$ of the time, $70.9 \%$ for cases that met the criteria, and $61.1 \%$ for cases that did not. For cases that did not meet the criteria, oncologists' concordance with CPGs ranged from $23.5 \%$ (case 9) to $100 \%$ (case 1) (Fig. 3a). For cases that met the criteria, oncologists' concordance with CPGs ranged from $17.7 \%$ (case 12) to $100 \%$ (case 18) (Fig. 3b). PCPs concurred with CPGs $60.6 \%$ of the time, $62.3 \%$ for cases that met the criteria, and $60.6 \%$ for cases that did not. For cases that did not meet the criteria, PCPs concordance with CPGs ranged from $18.2 \%$ (case 9) to $100 \%$ (case 1) (Fig. 3a). For cases that met the criteria, PCPs concordance with CPGs ranged from $18.2 \%$ (case 16) to $93.9 \%$ (case 18) (Fig. 3b).

Non-GCs had considerable discordance with CPGs for 7 of the 9 cases that met the CPG criteria implemented by ItRuns (cases 16, 12, 13, 10, 11, 17, and 15) for which GCs concurred with CPGs over $90 \%$ of the time (Table 1; Fig. 3). Oncologists and PCPs' level of consistency with
CPGs for these cases ranged from as high as $90.9 \%$ to as low as $17.7 \%$. The level of consistency with CPGs for all FHx cases by provider type can be viewed in Table 1. For one case that met the criteria - case 18 - providers had consistently high levels of concordance with CPGs $(98.7 \%$ overall). GCs and non-GCs had similar patterns of concordance for cases that did not meet the criteria; all provider types were frequently discordant with CPGs.

\section{Comparison of Concordance with CPGs between Provider Types}

To compare levels of concordance with CPGs, we ran several logistic regression analyses (Table 2). GCs had much higher concordance with CPGs for cases that met the criteria versus those that did not. When comparing GCs to non-GCs, GCs were 4 times more likely to concur with CPGs than non-GCs $(p<0.001)$. For cases that met the criteria only, GCs were 34 times more likely to concur 
Table 1. Concordance with CPGs by provider type

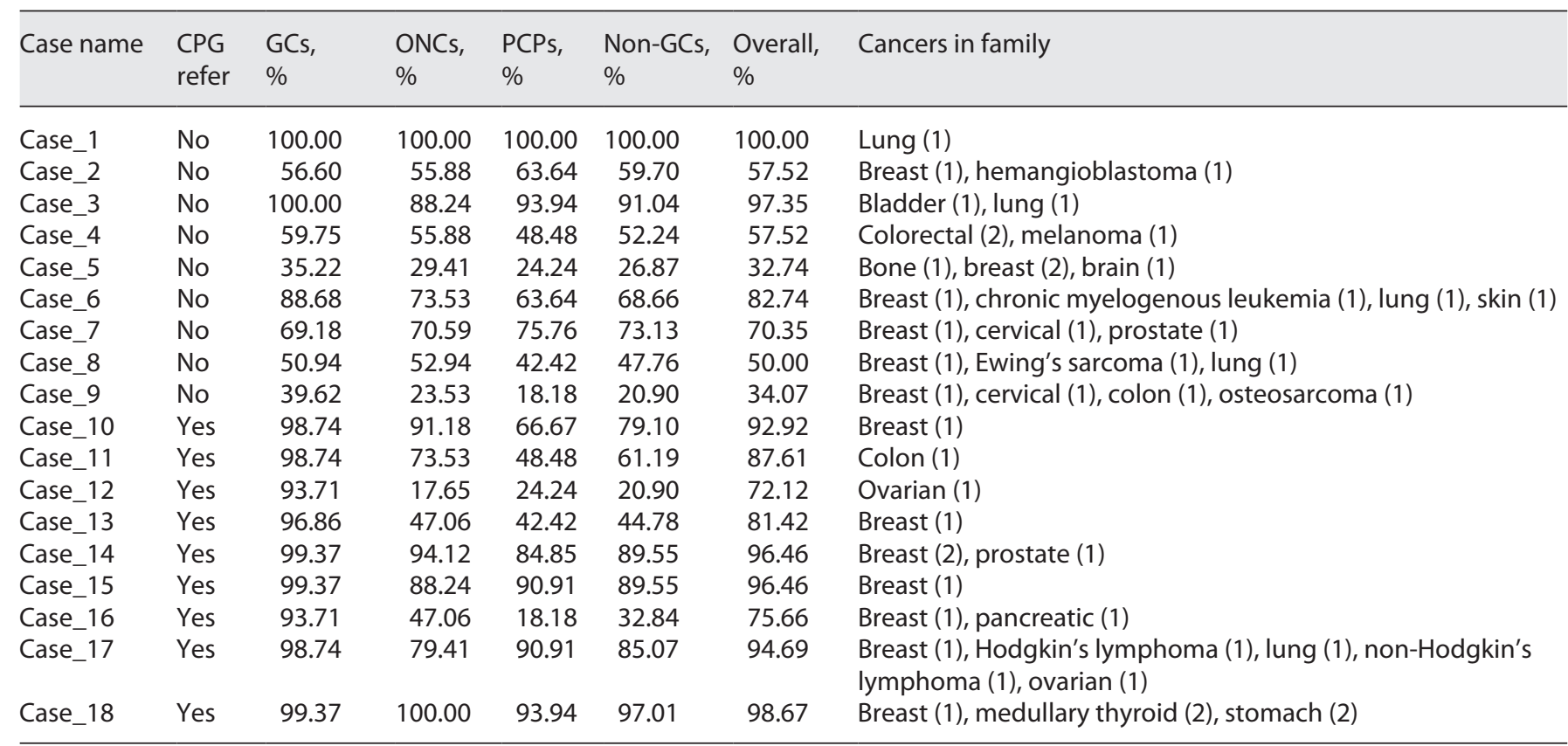

The first 9 rows correspond to FHx cases that did not meet criteria. The last 9 rows correspond to FHx cases that met the criteria. Additionally, the number of cancers and their type observed in each case are included in the Cancers column.

CPGs, clinical practice guidelines; ONCs, oncologists; GCs, genetic counselors; PCPs, primary care providers.

with CPGs than non-GCs $(p<0.001)$ and 1.5 times more likely $(p<0.01)$ for cases that did not meet the criteria. These results show that overall, GCs had statistically higher concordance with CPGs than non-GCs.

When comparing GCs to oncologists and to PCPs, GCs had much better odds of concordance with CPGs than oncologists or PCPs. Oncologists had marginally better odds than PCPs. Overall, oncologists and PCPs were $70 \%(p<0.001)$ and $79 \%$ less likely $(p<0.001)$ than GCs to concur with CPGs, respectively. For cases that met the criteria, oncologists and PCPs were 96\% ( $p<$ $0.001)$ and $98 \%(p<0.001)$ less likely to concur with CPGs than GCs, respectively. For cases that did not meet the criteria, oncologists and PCPs were 27\% $(p<0.034)$ and $36 \%(p<0.004)$ less likely to concur with CPGs than GCs, respectively.

\section{Discussion}

We distributed a survey to GCs and non-GCs with 18 FHx cases to explore the frequency with which provider decisions regarding referral for cancer genetic consultation were consistent with those of the web-based tool ItRuns. ItRuns used chatbot technology to collect family history and CPGs to assess risk. Half of the $18 \mathrm{FHx}$ cases met the CPG criteria, and half did not. The GC concordance with the ItRuns CPG criteria used was $82.2 \%$ overall and $97.6 \%$ for cases that met the CPG criteria. As expected, our results demonstrated that providers with specialized training in cancer genetics risk assessment and counseling made referral recommendations most consistent with ItRuns compared to other providers (Fig. 3).

Non-GCs were substantially discordant with CPGs for hallmark FHx cases such as 12, 13, 10, and 11, which GCs assessed correctly over $90 \%$ of the time (Table 1). Perhaps, the most noticeable discordance with CPGs for non-GCs was for the proband in case 12 who had an aunt diagnosed with ovarian cancer in her FHx. CPGs indicate that a first- or second-degree relative with ovarian cancer warrants referral regardless of age; oncologists had $17.7 \%$ and PCPs $24.2 \%$ concordance with CPGs versus $93.71 \%$ for GCs. Other classic CPG criteria involving Ashkenazi Jewish ancestry, close family members diagnosed with triple negative breast cancer before the age of 60 years, close family members diagnosed with breast cancer before 
Table 2. Coefficients, odds ratios, $95 \%$ confidence intervals, and $p$ values for logistic regression model fitted to survey data

\begin{tabular}{|c|c|c|c|c|}
\hline \multicolumn{5}{|c|}{ Comparing referral consistency of GCs to non-GCs (PCPs and ONCs) } \\
\hline & estimate & odds ratio & $95 \%$ conf int & $p$ value (sig) \\
\hline \multicolumn{5}{|l|}{ Overall } \\
\hline GC versus non-GC & 1.40 & 4.04 & $3.35-4.89$ & $<0.001^{*}$ \\
\hline \multicolumn{5}{|l|}{ Meets the criteria } \\
\hline GC versus non-GC & 3.53 & 34.00 & $22.52-52.92$ & $<0.001^{*}$ \\
\hline \multicolumn{5}{|c|}{ Does not meet the criteria } \\
\hline GC versus non-GC & 0.38 & 1.47 & $1.17-1.84$ & 0.001 \\
\hline \multicolumn{5}{|c|}{ Comparing referral consistency of ONCs and PCPs to GCs } \\
\hline & estimate & odds ratio & $95 \%$ conf int & $p$ value \\
\hline \multicolumn{5}{|l|}{ Overall } \\
\hline ONC versus GC & -1.22 & 0.30 & $0.23-0.37$ & $<0.001^{*}$ \\
\hline PCP versus GC & -1.58 & 0.21 & $0.16-0.26$ & $<0.001^{*}$ \\
\hline \multicolumn{5}{|l|}{ Meets the criteria } \\
\hline ONC versus GC & -3.26 & 0.04 & $0.02-0.06$ & $<0.001^{*}$ \\
\hline PCP versus GC & -3.81 & 0.02 & $0.01-0.04$ & $<0.001^{*}$ \\
\hline \multicolumn{5}{|c|}{ Does not meet the criteria } \\
\hline ONC versus GC & -0.32 & 0.73 & $0.54-0.98$ & 0.034 \\
\hline PCP versus GC & -0.45 & 0.64 & $0.47-0.86$ & 0.004 \\
\hline
\end{tabular}

the age of 50 years, and proband diagnosed with colorectal cancer before age 50 years were frequently under referred by non-GCs (Table 1).

Non-GCs' tendency to under refer patients who meet the CPG criteria is most likely related to the lack of cancer genetics-specific training and familiarity with hereditary cancer CPGs $[18,19]$. Obtaining patient $\mathrm{FHx}$, the time and effort required to apply CPGs for hereditary disease, and lack of reimbursement for time spent collecting and assessing FHx are barriers to identifying patients at increased risk for hereditary disease $[17,21,22]$. These barriers could be addressed by using technology such as ItRuns.

Discordance with the ItRuns referral recommendations could be, in part, related to the nature of CPGs and the specific ones used. CPGS are not validated tools; rather, they are educational documents curated by teams of experts based on empirical data and professional experience. Different organizations, such as ACMG and NCCN, approach the organization and dissemination of CPGs differently. The medium through which CPGs are published typically lags behind current practice, lacks standardization, and results in convoluted charts and tables that take considerable time and effort to decipher. Potentially noncomprehensive overly complex criteria limit
CPGs' effectiveness in accomplishing their primary purpose - to identify patients at risk of hereditary cancer based on their FHx.

Additionally, the CPG criteria suffer from an absolute cutoff problem; that is, some FHx cases might just miss the threshold for meeting criteria, but should still be referred for a cancer genetic consultation. For example, in case 9, the proband had 3 close relatives diagnosed with breast cancer, colon cancer, and osteosarcoma, all with age onsets after 56 years. If one of them had been diagnosed before the age of 45 years, this proband would have met the CPG criteria. GCs concurred with CPGs only $39.6 \%$ that case 9 should not be referred. This statistic might reflect a close-enough effect that most GCs did not concur with CPGs based on their clinical expertise and experience, and referred the patient for counseling and testing, even though case 9 technically missed the CPG age of the onset criteria. The CPGs are an important approximation of whether probands should be referred for counseling based on their FHx; however, they are not validated tools, may lag behind current knowledge, or may not be sensitive to detect a nuanced FHx that is suggestive of hereditary cancer. The ItRuns system is designed to match the CPG criteria exactly and therefore inherits some of these same limitations. Despite this shortcoming, GCs still very closely concurred with CPGs for
Ritchie et al. 
FHx cases, especially those that met the criteria, whereas oncologists and PCPs did not. A technological solution such as ItRuns that closely follows CPGs would support non-GCs in identifying patients that do meet the criteria and would otherwise be missed.

The fact that ItRuns and GCs had high concordance with CPGs for FHx cases that met the CPG criteria for referral is strong evidence that ItRuns can help identify patients at risk for hereditary cancer. The task of deciphering the CPG criteria and applying it to complex FHx data to facilitate referral to genetic counseling and testing could be enhanced using health information technology. Relying solely on human analysis and interpretation of CPGs for hereditary cancer risk assessment could lead to inconsistent referrals, or lack of referrals entirely, especially in non-GC medical settings. Data have shown that the identification of individuals with a high-penetrance cancer gene mutation and implementation of appropriate screening and risk reduction strategies can result in reduced morbidity and mortality [4-9]. The use of tools such as ItRuns can thus lead to population health benefits by helping nongenetics providers to make appropriate referrals for genetic counseling and testing. Additionally, ItRuns is free to use, which lowers barriers to access and promotes parity across all patient demographics with potential hereditary cancer risk.

Although risk assessment by genetic counselors can be considered the gold standard, the limited number of GCs argues that their expertise should be focused on providing counseling, disclosing genetic test results, and ensuring appropriate follow-up and disease management. An estimated $20 \%$ of primary care patients have a FHx that indicates an increased risk of developing hereditary cancer [30]. Clinical practice relies mostly on PCPs and oncologists for the initial screening of at-risk patients. Our results suggest that consistently deploying ItRuns across the primary care population could be as effective as GCs at identifying patients at risk of hereditary cancer.

The lack of concordance between non-GCs and CPGs for cases that met the criteria is a missed opportunity to refer patients for cancer genetics consultation that could result in preventive actions and improve health outcomes. Technology solutions such as ItRuns provide an opportunity to identify patients who need cancer genetic counseling without requiring the immediate time and attention of a provider to collect and assess FHx. The CPG criteria represented in the FHx cases in this study are wellpublished guidelines but are poorly adopted and inconsistently implemented on the front lines of health care, where non-GCs lack the time and training to collect and

Comparison of Cancer Family History

Risk Assessments assess FHx for hereditary cancer risk. Technology-based tools such as ItRuns can lead to greater numbers of at-risk individuals accessing genetic counseling, testing, and mutation-based interventions to improve health.

\section{Conclusion}

In this study, we compared GCs, oncologists, and PCPs' levels of concordance with the CPGs utilized by the tool ItRuns for referring patients at risk of hereditary cancer based on their FHx. GCs' concordance was high, especially for cases that met the CPG criteria, which supports its potential usefulness. The extent to which oncologists and PCPs did not concur with the CPG criteria compared to GCs represents an important limitation with the current strategies that are used to identify patients at increased risk for hereditary cancer. Technology solutions like ItRuns that make it simple to collect FHx and receive a CPG driven risk assessment have the potential to fill this gap and improve the identification and health of patients at risk for hereditary cancer. Additional research is needed to further validate ItRuns in larger samples of health-care providers. Future studies are also needed to evaluate the impact of broad application this tool has in a population-based setting. Tracking outcomes of referral and identification of individuals with a cancer gene mutation may also provide data to contribute to evidence-based adjustment of the existing CPG criteria.

\section{Statement of Ethics}

This study was reviewed and approved as exempt by the IRB at the Medical University of South Carolina. The survey included a study description and consent statement at the beginning of the survey.

\section{Conflict of Interest Statement}

Jordon B. Ritchie, Caitlin G. Allen, Lewis J. Frey, Alexander V. Alekseyenko, Brian Dean, Chanita Hughes Halbert, and Cecelia Bellcross had no conflicts to declare; Brandon M. Welch, Heath Morrison, and Joshua D. Schiffman were share holders and owners of ItRunsInMyFamily.com.

\section{Funding Sources}

This work was supported by the National Institutes of Health CA211786, CA239867, CA253576, and CA210962. 


\section{Author Contributions}

All authors contributed to the conception and design of this study, the acquisition of data, drafting the manuscript, and final approval prior to submission.

\section{Data Availability Statement}

All data generated or analyzed during this study are included in this article and/or its online supplementary material files. Further inquiries can be directed to the corresponding author.

\section{References}

1 Riley BD, Culver JO, Skrzynia C, Senter LA, Peters JA, Costalas JW, et al. Essential elements of genetic cancer risk assessment, counseling, and testing: updated recommendations of the National Society of Genetic Counselors. J Genet Couns. 2012 Apr;21(2):151-61.

2 Owens DK, Davidson KW, Krist AH, Barry MJ, Cabana M, et al, US Preventive Services Task Force. Risk assessment, genetic counseling, and genetic testing for BRCA-related cancer: US Preventive Services Task Force recommendation statement. JAMA. 2019 Aug 20;322(7):652-65. http://dx.doi.org/10.1001/jama.2019.10987.

3 Cohen SA, Bradbury A, Henderson V, Hoskins K, Bednar E, Arun BK. Genetic counseling and testing in a community setting: quality, access, and efficiency. Am Soc Clin Oncol Educ Book. 2019 Jan;39:e34-44.

4 Rebbeck TR, Kauff ND, Domchek SM. Metaanalysis of risk reduction estimates associated with risk-reducing salpingo-oophorectomy in BRCA1 or BRCA2 mutation carriers. J Natl Cancer Inst. 2009 Jan 21;101(2):80-7.

5 Xiao YL, Wang K, Liu Q, Li J, Zhang X, Li HY. Risk reduction and survival benefit of riskreducing salpingo-oophorectomy in hereditary breast cancer: meta-analysis and systematic review. Clin Breast Cancer. 2019 Feb; 19(1):e48-65.

6 Eleje GU, Eke AC, Ezebialu IU, Ikechebelu JI, Ugwu EO, Okonkwo OO. Risk-reducing bilateral salpingo-oophorectomy in women with BRCA1 or BRCA2 mutations. Cochrane Database Syst Rev. 2018 [cited 2021 Mar 23]; 8(8):CD012464.

7 Giannakeas V, Narod SA. The expected benefit of preventive mastectomy on breast cancer incidence and mortality in BRCA mutation carriers, by age at mastectomy. Breast Cancer Res Treat. 2018 Jan;167(1): 263-7.

8 De Felice F, Marchetti C, Musella A, Palaia I, Perniola G, Musio D, et al. Bilateral risk-reduction mastectomy in BRCA1 and BRCA2 mutation carriers: a meta-analysis. Ann Surg Oncol. 2015 Sep;22(9):2876-80.

9 Domchek SM, Friebel TM, Singer CF, Evans DG, Lynch HT, Isaacs C, et al. Association of risk-reducing surgery in BRCA1 or BRCA2 mutation carriers with cancer risk and mortality. JAMA. 2010 Sep 1;304(9):967-75.

10 van Riel E, van Dulmen S, Ausems MG. Who is being referred to cancer genetic counseling?
Characteristics of counselees and their referral. J Community Genet. 2012 Oct;3(4):26574.

11 Meyer LA, Anderson ME, Lacour RA, Suri A, Daniels MS, Urbauer DL, et al. Evaluating women with ovarian cancer for BRCA1 and BRCA2 mutations: missed opportunities. Obstet Gynecol. 2010 May;115(5):945-52.

12 Kne A, Zierhut H, Baldinger S, Swenson KK, Mink P, Veach PM, et al. Why is cancer genetic counseling underutilized by women identified as at risk for hereditary breast cancer? Patient perceptions of barriers following a referral letter. J Genet Couns. 2017 Aug; 26(4):697-715.

13 Evans O, Manchanda R. Population-based genetic testing for precision prevention. Cancer Prev Res. 2020 Aug;13(8):643-8.

14 Manchanda R, Blyuss O, Gaba F, Gordeev VS, Jacobs C, Burnell M, et al. Current detection rates and time-to-detection of all identifiable BRCA carriers in the Greater London population. J Med Genet. 2018 Aug;55(8):538-45.

15 Childers CP, Childers KK, Maggard-Gibbons $\mathrm{M}$, Macinko J. National estimates of genetic testing in women with a history of breast or ovarian cancer. J Clin Oncol. 2017 Dec 1; 35(34):3800-6.

16 Bero LA, Grilli R, Grimshaw JM, Harvey E, Oxman AD, Thomson MA. Closing the gap between research and practice: an overview of systematic reviews of interventions to promote the implementation of research findings. BMJ. 1998 Aug 15 [cited 2021 Feb 8]; 317(7156):465-8.

17 Welch BM, Wiley K, Pflieger L, Achiangia R, Baker K, Hughes-Halbert C, et al. Review and comparison of electronic patient-facing family health history tools. J Genet Couns. 2018 Apr;27(2):381-91.

18 Bellcross CA, Kolor K, Goddard KA, Coates RJ, Reyes M, Khoury MJ. Awareness and utilization of BRCA1/2 testing among US primary care physicians. Am J Prev Med. 2011 Jan 1;40(1):61-6.

19 Sussner KM, Jandorf L, Valdimarsdottir HB. Educational needs about cancer family history and genetic counseling for cancer risk among frontline healthcare clinicians in New York City. Genet Med. 2011 Sep;13(9):785-93.

20 Tyagi NK, Dhesy-Thind S. Clinical practice guidelines in breast cancer. Curr Oncol. 2018 Jun;25(Suppl 1):S151-60.
21 Vig HS, Armstrong J, Egleston BL, Mazar C, Toscano M, Bradbury AR, et al. Cancer genetic risk assessment and referral patterns in primary care. Genet Test Mol Biomarkers. 2009 Dec;13(6):735-41.

22 Doerr M, Teng K. Family history: still relevant in the genomics era. Cleve Clin J Med. 2012 May;79(5):331-6.

23 Welch BM, Allen CG, Ritchie JB, Morrison $\mathrm{H}$, Hughes-Halbert C, Schiffman JD. Using a chatbot to assess hereditary cancer risk. JCO Clin Cancer Inform. 2020 Sep;4:78793.

24 Ritchie JB, Bellcross C, Allen CG, Frey L, Morrison H, Schiffman JD, et al. Enabling patients to receive clinical practice guideline recommendations for hereditary cancer risk using chatbots, family history, Application Programming Interfaces (API), ontologies, and Owlready2: system description. J Med Internet Res. 2021 Apr. [under review].

25 Ritchie JB, Bellcross C, Allen CG, Frey L, Morrison H, Schiffman JD, et al. Evaluation and comparison of hereditary cancer guidelines in the population. Hered Cancer Clin Pract. 2021 Jul 17;19(1):31.

26 Ponathil A, Ozkan F, Welch B, Bertrand J, Chalil Madathil K. Family health history collected by virtual conversational agents: an empirical study to investigate the efficacy of this approach. J Genet Couns. 2020 Dec;29(6): 1081-92.

27 Ponathil A, Firat Ozkan N, Bertrand J, Welch B, Chalil Madathil K. New approaches to collecting family health history: a preliminary study investigating the efficacy of conversational systems to collect family health history. Proc Hum Factors Ergon Soc Annu Meet. 2018;62(1):277-81. http://dx.doi.org/10.1177/1541931218621064.

28 Drohan B, Roche CA, Cusack JC Jr, Hughes KS. Hereditary breast and ovarian cancer and other hereditary syndromes: using technology to identify carriers. Ann Surg Oncol. 2012 Jun;19(6):1732-7.

29 Ritchie JB, Allen CG, Morrison H, Nichols M, Lauzon SD, Schiffman JD, et al. Utilization of health information technology among cancer genetic counselors. Mol Genet Genomic Med. 2020 May 28;8:e1315.

30 Frezzo TM, Rubinstein WS, Dunham D, Ormond KE. The genetic family history as a risk assessment tool in internal medicine. Genet Med. 2003 Mar;5(2):84-91. 